\title{
RESEARCH REPORT \\ EFFECTIVENESS OF STRETCHING EXERCISES \\ IN COMPARISON TO KINESIO TAPING FOR THE CURE OF PLANTAR FASCIITIS PAIN
}

\begin{abstract}
\section{OBJECTIVE}

To determine the efficacy of stretching exercises verses kinesio taping for the cure of plantar fasciitis pain.
\end{abstract}

\section{BACKGROUND}

It is the apparent problem of foot that influences athletes. It transpires when rhythmic pressure is occurred on the heel as of a chronic or acute state. Physical therapists had apply many methods attempt to alleviate the warning signs of pain in plantar heel, as well as a variety of taping techniques for which there is slight accessible facts.

\section{METHOD}

Research was conducted at Outpatient Department of tertiary care hospital.

\section{INTERVENTIONS}

18 contestants amid plantar heel pain were hired from the ordinary community. Participants were randomly assigned into two groups. Group A participants were treated with kinesio taping, cold pack and ultrasound. Group B participants were treated with stretching, cold pack and ultrasound. The period of record for every participant was four weeks. No participants are missing to transcribe. Outcome assesses included pain in 'first-step' (evaluated on a Pain Scale from 1 to 10) plus the pain disability index Questionnaire.

\section{RESULTS}

Comprehensive information was acquired from eighteen participants. One-year record outcomes proved great improvement in every participant after application of stretching exercises of plantar fascia, with an chiefly lofty rate of upgrading in the actual group participants managed by the stretching exercises.

\section{CONCLUSION}

This study consists of the application of the stretching method which is the major element of management in chronic condition of plantar fasciitis. Continuing advantages of the stretching exercises include a noticeable reduction of pain and a lofty rate of achievement. These finding can give the health-care provider with an efficient, economical, and uncomplicated treatment method.

\section{KEY WORDS}

Plantar fasciitis, Plantar heel pain, repetitive micro trauma, plantar fascia, heel spur, nerve trapping, taping, stretching.

\author{
Syeda Rida Baqir \\ Physiotherapist \\ Physiotherapy Department \\ Ziauddin hospital \\ Dr_rida91@yahoo.com
}

Syed Abid Mehdi Kazmi

Associate Professor

Ziauddin College of Physical Therapy

syedabidmehdi@gmail.com

[Baqir SR, Kazmi SAM. Effectiveness of Stretching Exercises in Comparison to kinesio taping for the Cure of Plantar Fascitis Pain. Pak. j. rehabil. 2014;3(2):31-35] 


\section{INTRODUCTION}

Plantar fascia (P.F) is the broad fibrous structure. It is separated into 3 portions: lateral, medial, and central. The portion which is in the centreis the broadest one. It classified into five parts. These merge into the distal plantar side of the digits. The medial and lateral parts merge with the middle part as the route becomes further distal'.they deliver static support and shock absorption. Flat foot and elevated foot are the raised risk factors for plantar fasciitis. P.F is a degenerative condition that affects up to $10 \%$ of the common population ${ }^{2-3}$. Plantar fasciitis, seems that the obvious risk factor for inferior heel pain, is probably 11 to 15 percent common symptoms needing proficient be concerned among populations. Restriction sourced by alters in the structure which is the path physiological source of this condition, which develops pain and modification of gaitcycle ${ }^{2-3}$. A recent U.S study predictable that every year 1 million population visits for primary cure in different OPD and Clinics4. Incidence apparently hit the highest points between the 40 to 55 years of age among athletes. 1 It occurs as a result of recurring micro trauma leads to deterioration. ${ }^{3}$ Spur of heel and nerve trapping can be correlated with inflammation ${ }^{2}$. The most common symptom of P.F is throbbing pain in correlation to periods of rest. Pain that often limits regular activities ${ }^{4}$ and it affects women more often than men. The climacterics and the obese people are more prone to having it ${ }^{5}$.

On assessment, the contestant typically has maximum involvement of anteriomedial area of the calcaneus tenderness. Analysis is depending on the contestant's past record and objective assessment results. Contestants classically account a ongoing arrival of pain which is routinely shoddier with their initial footsteps after a phase of immobility. Associated parenthesis is uncommon. Contestants might account previous to the arrival of warning signs. Additional sources of pain are typically noticeable on the basis of medical record and objective examination.

Presently, no particular treatment has used to be effectual for the entire affected patients with plantar fasciitis. Now a day's many interventions are used including Electro physical agents ${ }^{5-6}$. manual therapy ${ }^{7-9}$ Stretching ${ }^{10-12}$, Taping ${ }^{13,14}$, Night splints, ${ }^{15}$ Steroid injections, Surgery, ${ }^{16-18}$ and Orthoses. ${ }^{15,19-22}$

Kinesio tape is a slim absorbent cotton material amid a remedial grade acrylic bonding agent. This absorbent tape is usually applied for N.Mand M.S.K circumstances; accordingly it is being supposed to be amazing in the spur of heel. Unevenly information be originated from contestants advantageous by kinesio tape on heel spur, still outcomes are combined. Management typically is preserved for four weeks previous to consequences might be predictable. Kinesio tape should be applied by an experienced practitioner, because incorrect applications can grounds additional concerns. Proper application of tape will last some days, still have washed. Kinesio tape is consisting of two versions thin and thick tape which can be applied in particular conditions.

The thin $2 \mathrm{~cm}$ is used by incised 4 narrow pieces of tape, somewhat extending the tape while placing it on the affected area ${ }^{23}$. Mostly these consequences in a luminary form appropriately on the hurting region. The $4-\mathrm{cm}$ tape is typically applicable by using two narrow pieces. One piece is somewhat elongated, applying from foot bottom up to the calf. The next piece is positioned beneath the foot; its chief function is to stay the elongated piece on its place. The tape can be extended up to $140 \%$. The purpose of plantar fasciitis taping is getting the patient to settle down their foot. If you have the foot very tight then before you stand up it will be painful because you are in excess of tightening the plantar fascia. To initiate an anchor is put at about the top of the foot where the plantar fascia ends. There is no pressure on this strip at all. It is figuratively just laid on the skin. The similar thing is done down the base of the foot over the heel. Be suspicious where the Achilles is as the tape can rub.

So we have an anchor at the base and an anchor at the top. This is where you necessitate relaxing the foot as the strips are used along the length of the plantar fascia. It can be easier to calculate up and rip the tape first instead of apply it to the foot still on the roll and rip it in area. Tape is applied from the center of the foot at the heel and fan superficial to the outside of the foot. A second strip is applied on the further side and a third in the axis of the foot. The strips of tape should starts from one anchor strip. Another strip is applied at the inner side of the foot which provides the taping a little bit more hold where it is required. The foot is still relaxed and the tape applied on the skin without any pressure then we again apply the anchors at the apex and base of the foot another time with no pressure in the tape. The last strip may be applied to give the medial arch of the foot even more hold. It sprint along the medial arch, around the heel and back crossways to the starting point.

After taping, the mobility still is maintained on muscle or joint. $^{3}$ it is an elastic tape it should be applied in prone position with knee joint $90 \%$ flexion. It give dynamic support, allow full ROM.

Stretching of plantar fascia can be done by a variety of methods: Towel stretch can be done by assembling on a solid plane amid affected one stretched out behind you. Round a towel at the base of foot and drags it towards your own maintained your knee position. Embrace the position for fifteen to thirty seconds after that calm down. Do again three times till the stretch made too simple, you can starts standing often center of attention on the plantar fascia itself. In Standing calf stretch can be done by in frontage of some barrier, fix your palms beside the barrier at on eye plane. Maintain the position of affected one behind the unaffected one frontward, and the base of your affected one place on the floor. Twirl your affected one somewhat inner as you leisurely bend forward towards the barrier in anticipation of you experience a stretch at the posterior aspect of your calf. Maintained for fifteen to thirty sec. Re-do three times. Perform those exercises regularly numerous moments a day. Until you can feel satisfaction, you will able to instigate stretching underside of your affected foot. Stretching of Plantar fascia: can be done by Stand amid the ball of affected one at the stairway. Cling to this position for fifteen to thirty seconds and afterward calm down Re-do three times.

Stretching is able to be perform by another method in which the therapist should be seated with involved foot traversed above own unaffected knee. After that, apply the already using hand over the area of his affected one and put patients foot backwards in the direction of his leg until he experience pressure at plantar fascia, We strongly emphasize stretching exercises, because they successfully reduce the probability of musculotendinous injuries to the foot and help to decrease soreness of the PF. It is momen- 
tous to carry out Stretching's numerous times daily, we Persist that all stretches be perform statically and that the preliminary hold position is 15 seconds. The stretch times rise by five seconds every day until a stretch time of one minute for apiece is attained. The prolonged stretching is requisite to trounce the stretch reflex. Also, the plantar fascia can be stretched with hand resistance by applying a force causing dorsiflexion of the great toe, embrace for up to one minute, re-do two or three times.

A Study conducted by Willis $\mathrm{B}^{24}$ showed that stretching showed a better decline in "pain at its worst" plus a pain specially in the sunrise.

\section{METHODOLOGY}

A randomized control-trial was conducted in 2014 and 2015. 18 participants amid inflammation of plantar fascia are contained in this research. They were arbitrarily splited by simple random sampling technique into two groups. Group A consist of 9 participants and Group B consist of 9 participants. Group A participants receiving muscle stretching with icepack and ultrasound, 3 sessions per week. All participants were counseled to do plantar fascia active stretch at home. And group B members receiving kinesio taping with icepack and ultrasound. All Participants were tracked up and directed by the similar physiotherapist in all treatment sessions. Ethical support for the experiment was expanded from ethics committee of the institution.

\section{Group Statistics}

Before stretching and tapping VAS (visual analog scale)

\begin{tabular}{|c|c|c|c|c|c|}
\hline & Group & $\mathbf{N}$ & Mean & $\begin{array}{l}\text { Std. } \\
\text { Deviation }\end{array}$ & $\begin{array}{l}\text { Std. } \\
\text { Error } \\
\text { Mean }\end{array}$ \\
\hline \multirow[t]{2}{*}{ BVAS } & Streching & 9 & 5.7778 & .97183 & .32394 \\
\hline & Tapping & 9 & 5.3333 & 1.22474 & .40825 \\
\hline
\end{tabular}

\section{After streching and tapping VAS(visual analog} scale)

\begin{tabular}{|r|l|l|l|l|}
\hline Group & N & Mean & $\begin{array}{l}\text { Std. } \\
\text { Deviation }\end{array}$ & $\begin{array}{l}\text { Std. } \\
\text { Error } \\
\text { Mean }\end{array}$ \\
\hline AVAS Stretching & 9 & 1.2222 & .97183 & .32394 \\
Tapping & 9 & 3.3333 & .70711 & .23570 \\
\hline
\end{tabular}

\section{PARTICIPANTS}

\section{INCLUSION CRITERIA}

Members were comprised if detected with inflammation of plantar fascia termed as:(1) Male and females b/w age group (40-55) years who have confirmed diagnosis of plantar fasciitis (2)Capable to bear the physical assessment and treatment methods (3) a symptom duration of over 4 weeks, As pain of heel is detected the preponderance of the instance ${ }^{25}$, we decided that we don't apply costly laboratory methods for analysis; consequently increasing our throughout results to usual medical protocol.

\section{EXCLUSION CRITERIA}

Members were prohibited from the study if client past record showed any inflammation, neurological, metabolic anomalies. Furthermore, excluded if they are: (1) Non-responsive to treatment (2) they have acute injury on the foot (3) They have tarsal tunnel syndrome (4) presence of foot or ankle fracture. Group members were not supported to initiate any other treatments throughout the assessment e.g.: (pain killers, night splints, corticosteroid injections and orthoses etc.).

\section{CLINICAL PROTOCOL}

Members were selected from confined population and treated at physiotherapy department in a tertiary care hospital. The distribution series was obscured from the investigator registered and evaluating members in consecutively numeral obscure conserved and clipped wrappings. To avoid insurrection of distribution order, person's name and D.O.B of the members was marked on the wrapper plus a videocassette prepared of the attached covering whereas stretching. Recommendation was advised to stretch daily until the transcribe scheduled time 2 weeks later. Members were not given any further training until the end of the trial.

Outcome evaluation was achieved in 14 days. Measures accumulated comprised gender, age, and participant informed period of warning signs. Main result assessment tools were pain felt while first standing after awaking up in the sunrise calculated by Pain Scale and the pain disability index survey. Both outcome calculations is self managed; though to reduce the investigator having control on member answers members finished result evaluations that were achieved earlier to every consultation.

\section{SAMPLE SIZE, DATA HANDLING AND ANALYSIS}

There is an eighteen sample size that are divided into 09 participants per group, estimated a priority, was depends upon the capability to perceive a least imperative disparity on the Pain Scale between groups. We conventionally disregard the further accuracy supplied by the covariate examination while guesstimate sample size between-participants by using a continuous product we were needed to search for evidence that provides the Independent researchers performed data entry to group allocation. Double means and standard deviations of the outcome in both group. The discrepancy between the two mean values and their particular variances states our evidence-based measure of effect which in turn leads to more accurate and perfect measures of effect.

An independent sample t-test was employed to establish but some differentiation among grouping in the time period which was given to them among results and transcribe scheduled times. Product information was evaluated via objective to manage and according to a pre-scheduled procedure. The results investigated to alter in pain (VAS Pain Scale), (pain disability index Questionnaire). We predict that the end results would be employed as the simply shown in investigation.

\section{RESULTS}

During the one year record assessment, whole statistics sets were attained from eighteen members (Group A and Group B consist of nine members), Group A was managed with the kinesio taping, icepack and ultrasound, and Group B was directed by the stretching treatment plan. 
Notice the pessimistic worth's replicate a lessening in pain scale results for every reliant assess. Subsequent achievement of the stretching course in all contestants by 4 weeks, major progress in the pain scale was note down ( $\mathrm{p}<$ $0.0001)$. This was examined from VAS Pain scale and the pain disability Index. The style of progress persist, and at the one-year transcribe stretching, icepack, ultrasound group had overall amounts of pain reduction compared with the kinesio taping, ice pack and ultrasound group. Actually, compare to the effects at the four-week record.

\section{DISCUSSION}

This is the first randomized control trial to compare taping and stretching technique for plantar heel pain. The difference between pre and post-treatment is shown on Visual Analogue Scale and pain disability index scale the result for members with plantar fasciitis has been calculated and is classically positive. The huge preponderance, around $90 \%$, contain declaration of the warnings within ten months. ${ }^{26}$ Stretching exercises, while essential to the majority of treatment practices, have seldom been assessed in segregation or else for their long-standing settlement. In assessing the facts from the latest research study, in addition to initial clinical examination, we note down generally optimistic reaction to the plantar fasciitis pain elongate. We consider as to pain scale at the four-week transcribe assessment each groups proved major upgrading as of baseline favorable of the group deal with the stretching program. At four weeks, every participant is specified the stretching procedure and progress persist. At one-year transcribe estimation; these modifications from baseline were important for every group $(p<0.0001)$. The potency of this research work is supported in its unique features, randomized plan plus continuing follow-up accessible at lyears. Additionally, accurate participant insertion criterion was used. If participants do not demonstrate the typical warning signs of plantar fasciitis, as well as soreness amid the preliminary strides in the sunrise, they were disqualified as from the research project. Additionally, participants amid constant indications intended for as a minimum 10 months be deliberately selected toward decrease outcome of a usual progress depending on the time, This is frequently noted in persons with acute plantar fasciitis. Until now research drawbacks do survive. At this point slow destruction rates of approximately $20 \%$ at the four-week study phase plus another time at the one-year transcribe estimation. This might have lead to modify outcomes. Another drawback is that the best point to accomplish effectual extend of plantar fascia is not approved. Advance studies are required to establish most favorable foot and ankle patterns that will more purify the plantar fascia-precise stretch. In support of participants amid chronic inflammation of plantar fascia, this research project supports the worth of stretching procedure. We think that it is a vital element of management, bigger to the conventional stretching of tendon. These consequences offer us amid an effectual, economical plus uncomplicated management procedure intended for management of persistent inflammation of plantar fascia moreover it demonstrate that stretching produces statistically important helpful effect on pain in contrast with kinesio taping.

\section{CONCLUSION}

At the conclusion of management, each patient treat with the stretching procedure were specified the stretching of plantar fascia procedure. Contestants were educated to execute work out inside method comparable to that utilized by unique crowd directed with the stretching procedure (i.e., thrice a day, amid the initial extend made earlier than the early step was taken in the sunrise, the contestant maintained the stretch do ten repetitions and again do its ten repetitions $s$ in each session). They were appealed to do the stretching procedure for as a minimum (IV) weeks and afterward while pain occurred. In addition to, the participants were optimistic to persist to apply and make available to them in whole management course.

\section{REFERENCES}

[1] Gray R Gray's Anatomy. Philadelphia: W.B. Saunders Co; 19 0:612.

[2] Zanon RG, Kundrat A, Imamura M. Ultra-somcontínuo no tratamento da fasciitis plantar crônica. Acta Ortop Bras. 2006;1 14:137-40.

[3] Roxas M. Plantar Fasciitis: diagnosis and therapeutic deliberations. Alt Medical Revision. 2005;10(2):83-93.

[4] Badlissi F, Dunn JE, Link CL, Keysor JJ, McKinley JB, Felson DT: Foot M.S.K disorders, ache, and foot-related functional restrictions in older persons. J Am Geriatr Soc. Jun 2005;53(6):1029-33.

[5] Imamura $M$, Carvalho Junior $A E$, Fernandez TD, Leivas TP, Kaziyama HH, Ferraz TB. Fascite Plantar: estudo comparativo. Rev Bras Ortop. 1996; 31:561-6.

[6] Kiritsi O, Tsitas K, Malliaropoulos N, Mikroulis G. Ultrasonographic assessment of plantar fasciitis after low-level laser therapy: results of a double-blind, randomized, placebo-controlled trial. Lasers Med Sci. 2010 25:275-81.

[7] Stratton M, McPoil TG, Cornwall MW, Patrick K. Use of electrical stimulation of very small-frequency for the treatment of plantar fasciitis's Am Podiatric Med Assoc. 2009:99(6):481-88.

[8] Cleland JA, Abbott JH, Kidd MO. Manual physical therapy and exercise versus electro physical agents and exercise in the management of plantar heel pain: a multicenter randomized clinical trial. J Orthop Sports Phys Ther. 2009:39(8):573-85.

[9] Young B, Walker MJ, Strunce J, Boyles R. A combined treatment approach emphasizing impairment-based manual physical therapy for plantar heel pain: a case series. J Orthop Sports Phys Ther. 2004:34(1 1):725-733.

[10] DiGiovanni BF, Nawoczenski DA, Lintel ME, Tissue-specific plantar fascia-stretching exercise enhances outcomes in patients with constant heel ache. A prospective, randomized study. J Bone Joint Surge Am. 2003;85-A:1270-1277.

[11] Radford JA, Landorf KB, Buchbinder R, Cook C. Effectiveness of the stretching of calf muscle for the short-term treatment of plantar heel pain: a randomized trial. BMC Musculoskelet Disord. 2007;8:36.

[12] O'Sullivan K, Kennedy N , O'Neill E, Ni Mhainin U . The effect of low-Dye taping on rear foot motion and plantar pressure during the stance phase of gait. 
BMC Musculoskelet Disord. 2008;9111.

[13] Radford J, Landorf KB, Buchbinder R, Cook C. Effectiveness of low-Dye taping for the short-term treatment of plantar heel pain: a randomized trial. BMC Musculoskelet Disord. Aug 2006;7:64.

[14] Martin JE, Hosch JC, Go fort WP, Muff RT, Lynch DM, Odom RD. Mechanical treatment of plantar fasciitis. A prospective study. J Am Podiatric Med Assoc.2001:91:55-62.

[15] Tatli YZ, Kapasi S. The real risks of steroid injection for inflammation of plantar fascia, with an appraisal of conventional treatment. Curr Rev Musculoskeletal Med.2009;23-9.

[16] Liden B, Simmons M, Landsman AS. A retrospective investigation of 22 participants treated with percutaneous radiofrequency nerve ablation for prolonged moderate to severe heel pain associated with inflammation of plantar fascia. J Foot Ankle Surg. Nov-Dec 2009:48(6):642-47.

[17] Miyamoto W, Takao M, Uchio Y. Calcaneal osteotomy for the management of plantar fasciitis. Arch Orthop Trauma Surg. Feb 2010; 130(2):151-54.

[18] Roos E, Engstrom M, Soderberg B. Foot orthoses for the treatment of plantar fasciitis. Foot Ankle Int. Aug 2006;27(8) 606-11.

[19] Baldassin V, Gomes CR, Beraldo PS. Effectiveness of manufactured and modified foot orthoses made from low-cost foam for non complicated plantar fasciitis: a randomized controlled trial. Arch Phys Med Rehabil. April 2009:90(4):701-06.

[20] Landorf KB, Keenan AM, Herbert RD. Effectiveness of different varieties of foot orthoses for the treatment of plantar fasciitis's Am Podiatr Med Assoc. Nov-Dec 2004:94(6):542-49.

[21] Landorf KB, Keenan AM, Herbert RD. Effectiveness of foot orthoses to care for plantar fasciitis: a randomized control trial. Arch Intern Med. Jun 2006;166(12):1305-10.

[22] American College of Foot and Ankle Consultants. The analysis and management of heel pain. J Foot Ankle Surg. 2001;40:329-40.

[23] Chen YL. The Technique of Kinesio taping. Taipei (Taiwan): The Community Health and Sports Association of the Republic of China, 1995.

[24] Willis B, Lopez A, Perez A, Sheridan L, Kalish SR. Pain Scale for Plantar Fasciitis. The Foot and Ankle Online Journal. May 2009;2(5):3

[25] Davis PF, Severud E, Baxter DE. Painful heel syndrome: results of non operative management. Foot Ankle Int. Oct 1994;15(10):531-5

[26] Pfeffer G, Bacchetti P, Deland J, et al. Comparison of custom and manufactured orthoses in the initial treatment of proximal inflammation of plantar fascia. Foot Ankle Int 1999;20:214-21 